\title{
Which idea is better with regard to immune response? Opioid anesthesia or opioid free anesthesia
}

\author{
Barbara Lisowska (D) \\ Jakub Jakubiak ${ }^{2}$ \\ Katarzyna Siewruk $\mathbb{D}^{3}$ \\ Maria Sady (D) $^{3}$ \\ Dariusz Kosson ${ }^{4}$

\begin{abstract}
'Department Anesthesiology and Intensive Medical Care, National Geriatrics, Rheumatology and Rehabilitation Institute, Warsaw 02-637, Poland; ${ }^{2}$ Department of Anesthesiology and Intensive Care, John Paul II Western Hospital, Grodzisk Mazowiecki 05-825, Poland; ${ }^{3}$ Faculty of Veterinary Medicine, Department of Large Animal Diseases with Clinic, Warsaw University of Life Sciences, Warsaw 02-797, Poland; ${ }^{4}$ Department of Anaesthesiology and Intensive Care, Division of Teaching, Medical University of Warsaw, Warsaw 02-005, Poland
\end{abstract}

This article was published in the following Dove Press journal: Journal of Inflammation Research

\begin{abstract}
The stress of surgery is characterized by an inflammatory response with immune suppression resulting from many factors, including the type of surgery and the kind of anesthesia, linked with the drugs that are used and the underlying disease of the patient. The trauma of surgery triggers a cascade of reactions involving the immune response and nociception. As strong analgesics, opioids provide the analgesic component of general anesthesia with bi-directional effect on the immune system. Opioids influence almost all aspects of the immune response in regards to leukocytes, macrophages, mast cells, lymphocytes, and NK cells. The suppressive effect of opioids on the immune system is limiting their use, especially in patients with impaired immune response, so the possibility of using multimodal anesthesia without opioids, known as opioid-free anesthesia (OFA), is gaining more and more sympathizers. The idea of OFA is to eliminate opioid analgesia in the treatment of acute pain and to replace it with drugs from other groups that are assumed to have a comparable analgesic effect without affecting the immune system. Here, we present a review on the impact of anesthesia, with and without the use of opioids, on the immune response to surgical stress.
\end{abstract}

Keywords: opioids, opioid-free anesthesia, immune response, surgical stress

\section{Introduction}

The function of the immune system consists of distinguishing between self-antigens and foreign antigens, which in turn protects the organism from the many potentially deadly antigens by triggering their elimination.

When speaking about the mechanisms of immune responses one should mention the innate and the acquired immunity. The main differences between them concern the duration of the reactions and the type of the immune cells involved in them. A characteristic feature of the innate response is its short reaction time and the participation of leukocytes and dendritic cells. Macrophages, formed from monocytes, together with dendritic cells belong to the group of antigen-presenting cells. Innate immunity is the first stage response to many pathogens and it also plays an important role in the development of autoimmune diseases, such as demyelinating syndromes, connective tissue diseases, and atherosclerosis. ${ }^{1}$

In contrast, lymphocytes play a key role in the acquired response and two types of functional immune responses can be identified: humoral immune response, sometimes called antibody-mediated immune response, where the antibodies are secreted by $\mathrm{B}$ lymphocytes, and cell-mediated immune responses involving $\mathrm{T}$ lymphocytes

\footnotetext{
Correspondence: Barbara Lisowska

Tel +48-22-755-920l

$\mathrm{Fax}+48-22-755-9109$

Email blisowska19@gmail.com
}

Journal of Inflammation Research 2020:13 859-869 
which are activated by peptides from the antigens displayed on the antigen-presenting cells. ${ }^{2-4}$

The next important difference between humoral and cellular immunity concerns the places of response. The humoral immunity occurs outside the infected cells and while cellular immunity takes place inside cells.

Among the T-lymphocytes, several subpopulations can be mentioned, among them helper (Th helper, CD4), regulatory (Treg CD4 $+\mathrm{CD} 25)$, and cytotoxic $\left(\mathrm{T}_{\mathrm{C}}\right.$ cytotoxic CD8) T-cells, which regulate the immune response.

The trauma of surgery triggers a cascade of reactions involving the immune response and nociception, which attempts to minimize its effects and maintain a homeostasis. The relationship between the inflammatory response and the nociception is maintained by the release of endorphin, enkephalin, and dynorphin from leukocytes $\beta$ by stimulation of the CRF and IL-1 $\beta$. The effect is similar to the one achieved as a result of stimulation of adrenergic fibers by noradrenaline (NA), which causes the release of endorphins through the receptors on sensory neurons, which in turn inhibits the conductivity of nociception at the site of the injury. ${ }^{5,6}$

To paraphrase, it can be said that the cascade of processes involved in the immune response is activated and then ended by the start and then the curbing of the inflammation.

The extent of surgical trauma depends on three basic elements: the general condition of the patient, the scope and duration of the surgery, and the kind of anesthesia in terms of the used drugs and the technique. The next important factor in surgical stress is pain, the intensity of which is reflected in the CNS response, especially within the hypothalamic-pituitary-adrenal (HPA) axis since the HPA stimulation by nociceptive stimuli causes a decrease in the activity of the NK cells and upsets the CD4/CD8 ratio. ${ }^{7}$

The NKT (natural killer T cell) cells are lymphocytes that have the ability to recognize the CD1d molecules that form a lipid antigen complex, which is not recognized by classic lymphocytes. Another characteristic feature of the NKT cells is their ability to be immediately active.

The NKT cells are known as cells belonging to both the innate and the acquired response. NKT like cytotoxic $\mathrm{T}$ lymphocytes and activated macrophages are involved in cellular immunity. Among these,

NK cells play a critical role in limiting cancer cells development and progression and are involved in cellular immunity after surgery. A suppression of the NKT cells may increase the possibility of infection and postoperative tumor spread.
By releasing many cytokines, the NKT cells can stimulate the activity of other cells involved in the immune response.

Surgical stress causes a decrease in circulating NKT cells through the activation of the programmed death-1 (PD-1) receptor and the programmed death of its ligand1 (PD-L1), whose expression increases on the surface of stimulated T and B lymphocytes. ${ }^{8}$

Surgical stress may also contribute to the weakening of lymphocyte activity and to changes in the $\mathrm{Th} 1 / \mathrm{Th} 2$ ratio in favor of the Th2 lymphocytes that participate in humoral response. ${ }^{9,10}$

Excessive inflammation or multiple immune cell suppression may cause immune response disorder during surgical stress.

Opioids are strong analgesics used in the treatment of acute and chronic pain. Analgesics from this group are very effective, but they cause many side effects, among which their bi-directional action on the immune system has been arousing the interest of researchers and clinicians for years. At the base of this relation are opioid receptors, with affinity for both endogenous and exogenous opioids, distributed on the cells of the immune system. ${ }^{11}$ Recent investigations confirmed the interrelationship between the immune system and the opioids, both in terms of stimulatory and suppressive effects, but this is still not clearly understood. For this reason, researchers and clinicians are interested in the possibility of using multimodal anesthesia without opioids.

In this review, we discuss the influence of either opioids or non-opioid analgesics used under opioid-free anesthesia (OFA) on the immune response to surgical stress as described in scientific literature and official reports. We conducted a systematic search in MEDLINE for biomedical literature, as well as in multidisciplinary databases, such as Scopus and Web of Science, using the following combined terms: opioids, anesthesia, acute pain, opioids free anesthesia, drugs used in OFA, factors of immune response, stress surgery.

\section{The Influence of the Opioids Used in Anesthesia on the Immune Response in Relation to Surgical Trauma}

During anesthesia, opioids belonging to the fentanyl group (sufentanil and remifentanil) produce powerful analgesia of acute pain.

Fentanyl (FN) is the reference opioid in the group of synthetic opioids whose potency is 100 times greater than 
morphine. Like other opioids, it demonstrates analgesic and sedative effects. Both the onset of its action and the severity of its side effects, manifesting in the form of respiratory and circulatory depression, depend on its dose and way of administration.

The metabolism of fentanyl is mediated by cytochrome P450 and its CYP3A4 isoenzyme. These enzymes may have an essential influence on its effectiveness and this fact should be stressed, as the same isoenzyme participates in the metabolism of other drugs, eg, psychotropic drugs and benzodiazepines, or is blocked by other drugs, eg, antifungal and anti-arrhythmic drugs, which constitutes a possibility for a very risky interaction. ${ }^{12,13}$

Different opioids or different methods of opioid administrations show various effects on the immune system: immunosuppressive, immunostimulatory, or dual effect.

Sufentanil (sFN) is a high potency synthetic fentanyl analog; with about 5-10 times stronger, faster, and shorter action, which is due to its very good solubility in fat, and thus an easy penetration of the blood-brain barrier. Like fentanyl, sufentanil is also metabolized by the CYP3A4 isoenzyme, so its risk of interaction is similar to fentanyl. Unlike the mentioned opioids, remifentanil $(\mathrm{rFN})$ is an opioid of which $95 \%$ is metabolized in the plasma and in tissues by non-specific blood and tissue esterases.

Therefore, remifentanil has the highest margin of safety (LD50/ED50). Its potency is similar to the potency of fentanyl. ${ }^{14,15}$

Opioids interact via mu-, delta-, and kappa receptors among which the $\mu$ receptor deserves special attention because it is a preferential receptor for exogenous opioids.

Like other opioids and opiates, the drugs from this group: fentanyl, remifentanil, and sufentanil, are ligands of the $\mu$ opioid receptors (MOP, $\mu$-opioid peptide) belonging to one of three types of membrane receptors with different affinities for exogenous and endogenous opioids. Opioid receptors belong to the G-protein-coupled receptor family (GPCRs). Following the binding of the opioid, there is a change in the configuration of the receptor, which after attaching to the $G$ protein activates intracellular processes with inhibition of cAMP synthesis and a change in the configuration of an ionic channel that ultimately leads to inhibition of the release of neurotransmitters and the transmission of nociceptive signals. ${ }^{16}$

Results of scientific research as well as by clinical observations of patients confirming different analgesic responses and severity of side effects made it possible to isolate the $\mu$ receptor subtypes: $\mu$ opioid type 1 (MOP1) and $\mu$ opioid type 2 (MOP2) having a diverse sequence of polypeptides associated with a nucleotide polymorphism in the MOR-1 gene coding for the receptor proteins. ${ }^{17,18}$ Differences between these types of receptors refer to their sensitivity for their selective antagonists, such as naloxazone (the hydrazone derivative of naloxone) and naloxone. ${ }^{19}$

The $\mu$ receptors transmit their activity through pre- and postsynaptic neurons. The effect of stimulation of the $\mu$ receptors is similar with respect to the opioids, but different with respect to their action at the level of the neuron. And so, in the presynaptic part, there occurs a calcium channel blockage, while in the postsynaptic part a hyperpolarization occurs due to the influx of potassium ions into the cells. To sum up, the MOR receptors inhibit $\mathrm{Ca}^{++}$influx through calcium channel blockage at presynaptic terminals, and postsynaptically inhibit the flow of $\mathrm{K}^{+}$ions through the potassium channels, which reduces the frequency of pain impulses. Studies conducted on isolated tissues of the spinal cord of rats confirmed two opioid attachment points, whose synergistic effect results in an analgesic effect. ${ }^{20}$

Studies presented in recent years confirmed high expression of miRNAs in the cells of the immune system and in the CNS, the main sites for the opioid activity, and for the transformation of nociceptive stimuli. ${ }^{21-23}$

MicroRNAs (miRNAs) belong to the group of singlestranded non-coding RNAs that regulate gene expression at transcriptional and post-transcriptional levels. MiRNAs are made up of short fragments of 21-23 nucleotides. Their regulation is usually negative, as after binding to the target gene's mRNA the micro-RNA accelerates its degradation or inhibits the process of its translation, depending on the degree of its complementarity with the gene. $^{24}$

Silencing of the immune response by the expression of miR-146a and miR-21 on the T lymphocytes and PBMCs is an example of a negative feedback, whereas the expression of miR-155 on the $\mathrm{T}$ and $\mathrm{B}$ lymphocytes promotes activation of the immune response. ${ }^{25}$

The relationship between the miRNAs and the immune response is confirmed by numerous studies showing the presence of miRNAs in the cells of the immune system. For example, the study by Liston et al confirmed the expression of miR-29 family in the $\mathrm{T}$ and $\mathrm{B}$ lymphocytes and dendritic cells. ${ }^{26}$

Zhang et al in turn isolated 108 miRNAs present in peripheral blood mononucleated cells (PBMCs) obtained from healthy volunteers. ${ }^{27}$ 
Additionally, based on the obtained results, the researchers determined 10 abundantly expressed miRNAs in the studied material, including miR-223, mi-16, and mi-150, or miRNAs with confirmed involvement in the modification of the innate response associated with a change in their expression in B lymphocytes, monocytes, and neutrophils. ${ }^{28}$

The involvement of miR-146, miR-155, and miR-223 in the regulation of innate responses and the association of miR-155 and miR-181a with responses from the $\mathrm{T}$ and B lymphocytes has also been demonstrated. ${ }^{29}$ Studies have shown that miRNAs are involved in the regulation of both the analgesia-related and the side effects-related opioid activities. $^{23}$

For example, studies conducted by Zheng $\mathrm{H}$. on an animal model showed a reduction of the miRNA-190 expression in the hippocampal neurons due to chronic fentanyl administration. ${ }^{30,31}$ The negative regulation of the miR-134 expression of the MOR1 receptor at the level of the spinal cord neurons following a chronic inflammatory pain stimulation in laboratory animals has also been confirmed. ${ }^{32}$

Opioids are responsible for the modulation of the immune response due to a release of cytokines from the immune cells in response to stimulation of the opioid receptors. The released cytokines exhibit pleiotropic autopara- and auto-endocrine activity in both types of immune responses. It has been shown that opioids can induce the IL-4 release by T lymphocytes, while both buprenorphine and morphine reduce the level of IL-4 protein synthesis, which would confirm both the anti-inflammatory and the pro-inflammatory effects of the opioids. ${ }^{33}$

Interleukin -1 (IL-1) belongs to the pro-inflammatory cytokines released from many cells, such as the cells of central nervous system, monocytes, macrophages, dendritic cells, and NK cells. Opioids can reduce the plasma levels of IL-1. Like opioids, ketamine and propofol can also reduce plasma IL- $1 .{ }^{34}$

In comparative studies of remifentanil vs fentanyl, it was found that in response to surgical stress remifentanil caused a greater than FN reduction in IL- $6 .{ }^{35}$ Research conducted by Von Drossow et al showed that remifentanil more than FN attenuated the inflammatory response in patients after cardiac surgery. ${ }^{36}$

The presence of opioid receptors on $\mathrm{T}$ cells is one more evidence of the immunomodulatory effect of opioids on the differentiation of the cellular response. ${ }^{21}$ For example, a stimulation of the kappa receptors on macrophages increases the synthesis of reactive oxygen species and the IL-1. ${ }^{37}$
Opioids influence almost all aspects of the immune response, both innate and acquired, in relation to leukocytes, macrophages, mast cells, lymphocytes, and NK cells, although the results of the studies presented by Jakobs et al did not confirm the effect of fentanyl on the number of circulating $\mathrm{B}$ and $\mathrm{T}$ lymphocytes and on the production of superoxide by the PMNCs. ${ }^{38}$

The presence of MOR and $\delta$ and $\kappa$ receptors has been confirmed on the surface of human dendritic cells (DCs) involved in both the cellular (inducing $\mathrm{T}$ cell proliferation and presentation of antigens to $\mathrm{T}$ lymphocytes) and humoral (at the level of lymphoid papules, they participate in the presentation of antigen to B lymphocytes) response.

The presence of MOR receptor agonists on activated dendritic cells (DCs) provoked them to increase the synthesis of IL-10 with a decrease in proinflammatory cytokines IL-12 and IL-23. ${ }^{39}$ The cytokines IL-12 and IL-23 are involved with carcinogens. The IL-12 has a strong antitumor effect by inhibiting angiogenesis and by stimulating the synthesis of anti-proliferative IFN- $\gamma$ and TNF, as well as a positive effect on the T and NK lymphocyte cytotoxicity and the tumor infiltration by CD8 lymphocytes, whereas the IL-23 reduces the CD8 infiltration and promotes angiogenesis. ${ }^{40}$

With respect to tumors, opioids can modulate their development potential by affecting both their proliferation and apoptosis. On the one hand, confirmed MOR overexpression, and thus the opioid effect, is associated with tumor development and metastasis. The published data seem to confirm the diversity of opioid activity depending on the type of cancer, shown, for example, by the results of the research concerning lung cancer, and bowel and pancreatic cancer. ${ }^{41-43}$

The research conducted by Yardeni et al evaluated the effect of different doses of fentanyl on pro- and antiinflammatory cytokine production and on NK cell cytotoxicity in perioperative patients. The obtained results showed the influence of the dose of FN on the secretion of IL-1 $\beta$, IL-2, IL-6, and IL-10. Significant attenuation of the IL-1 $\beta$ and IL-6 cytokine was noticed in groups of patients taking high $(70-100 \mu \mathrm{g} / \mathrm{kg})$ and medium (23-30 $\mu \mathrm{g} / \mathrm{kg}$ ) doses, while doses of 2-4 $\mu \mathrm{g} / \mathrm{kg}$ lacked significant postoperative effect on the concentration of the cytokines tested. Dose-independent suppression by opioids was found for the IL-2 and for the NK cells. ${ }^{44}$

Beilin et al, in turn, assessed the effect of intraoperative administration of doses of FN defined as high (75-100 $\mu \mathrm{g} / \mathrm{kg})$ and small $(1-5 \mu \mathrm{g} / \mathrm{kg})$ on NK cells in the presence 
of IL-2 or INF $\alpha$ and $\beta$ because these cytokines exert a positive effect on the NK cytotoxicity. The obtained results confirmed the postoperative decline of the NK cytotoxicity at 24 hours regardless of the FN dose. In the group of patients receiving a low dose of FN, the activity of the NK cells returned to the ground level at $48 \mathrm{hrs}$, while in the patients receiving a high dose, the NK activity remained at the lowered level.

In addition, this study showed that the presence of cytokines improved the NK cytotoxicity, and IL-2 contributed especially to the reversal of the NK suppression. ${ }^{45}$ The results of these studies, showing the negative effect of high doses of fentanyl on the NK activity should force us to verify the intraoperative dosing of FN, especially in cancer patients. Inhibition of the NK activity and T cell proliferation by synthetic opioids should be taken into account while making decisions about their administration. ${ }^{46}$

\section{Key Points}

The presence of MOR on the surface of immune cells confirms opioids participation in cellular and humoral response.

Remifentanil more than fentanyl attenuated the inflammatory response.

With respect to tumors, opioids can modulate their development potential by affecting both their proliferation and apoptosis. The diversity of opioid activity depends on the type of cancer.

The synthetic opioids cause depression of the activity of the natural killer (NK) cells. Negative effect of high doses of fentanyl on the NK activity should force the doctors to verify the intraoperative dosing of FN, especially in cancer patients.

The suppressive effect of opioids on the immune response is an element limiting their use, especially in patients with impaired immune response, so the possibility of using multimodal anesthesia without opioids, known as opioid-free anesthesia (OFA), is gaining more and more sympathizers. This can undermine the paradigm that has existed for years, about the necessity of using opioids during general anesthesia.

\section{Is Opioid-Free Anesthesia a Good or Bad Idea in Relation to the Immune System?}

The concept of OFA is to eliminate opioid analgesia in the treatment of acute pain and to replace it with drugs from other groups that are assumed to have a comparable analgesic effect, but without affecting the immune system. The perioperative analgesic effect of OFA is achieved through the use of non-opioid analgesics, NSAIDs, and coanalgesics.

It should be clearly emphasized here that the drugs used in OFA, such as ketamine and lidocaine, may also be used with opioids. Intravenous and inhalational anesthetics, as well as regional anesthesia, may also be used as permanent elements of the OFA or opioid anesthesia.

Obese patients, patients with opioid addiction as a result of chronic use, and oncological patients are part of a group where OFA plays a particular role. Among the contraindications to the use of OFA are circulatory insufficiency, cardiac arrhythmias (especially bradyarrhythmias), hypovolemia, shock, unstable coronary artery disease, autonomic neuropathy with orthostatic hypotension, and history of allergic reactions to the proposed drugs. ${ }^{47,48}$

Therefore, since OFA is used in a significant group of patients, it is reasonable to analyze the effects of the drugs used in OFA with respect to their analgesic effect and their impact on the immune system. The section below presents the effects of the drugs used in OFA on the immune system and may be interpreted as an attempt to answer the question whether the OFA is better than the opioid anesthesia in terms of the immune system.

\section{Ketamine}

Ketamine belongs to the recognized OFA anesthetics because it has better hemodynamic stability than other intravenous anesthetics. Ketamine is an antagonist of the glutaminergic ionotropic NMDA receptor.

As was shown in human and animal studies, ketamine exerts immunomodulatory and anti-inflammatory effects. The anti-inflammatory effect of ketamine is manifested by inhibition of both the humoral and the cellular responses. In contrast, its immunosuppressive effect consists of inhibiting lymphocyte proliferation, limiting the NK cell activity and abundance, as well as the phagocytic capacity of neutrophils. Ketamine also impairs the function of macrophages and weakens the adhesion of neutrophils and leukocytes to the substratum. ${ }^{49}$ Noteworthy are the results of the studies conducted by Beilin et al, in which the authors demonstrated the effect of low doses of ketamine, $0.15 \mathrm{mg} / \mathrm{kg}$ administered before the induction of anesthesia, on the concentration of the cytokines IL-1 $\beta$, IL-2, IL-6, and $\mathrm{TNF} \alpha$, the cytotoxicity of neutrophils, and the mitogenstimulated lymphocyte proliferation. ${ }^{50}$ A single dose of 
ketamine inhibited the increase in IL- 6 and TNF $\alpha$ at 4 hours after surgery, while the subsequent determinations showed an increase in the concentration of these cytokines similar to the control group. The inhibitory effects of ketamine on the concentration of proinflammatory cytokines have also been confirmed by the studies reported by other authors. ${ }^{51-53}$ However, studies led by $\mathrm{Li}$ et al have shown an increase in the pro-inflammatory cytokines IL- 6 and IL- $1 \beta$ in mouse hippocampal cells irrespective of the time and method of administration, while the concentration of TNF $\alpha$ measured in the study increased under the influence of a single dose and decreased under the influence of a chronic supply, which may indicate the participation of ketamine in neuroinflammatory processes. ${ }^{54}$

Another noteworthy fact is that the ketamine-induced immunosuppression that is associated with a decrease in the NK cell activity and lymphocyte counts is reflected as a greater risk of the growth of cancer cells, which may constitute a significant contraindication to long-term use of ketamine in cancer patients. ${ }^{55}$

Ketamine plays a significant role in analgesic management in both acute and chronic pain. Supplying ketamine makes it possible to reduce the need of administration of opioids while reducing the intensity of pain by inhibiting the flow of nociceptive stimuli into the CNS. $^{56-58}$

With regard to the inflammatory response, ketamine reduces the adhesion of neutrophils and leukocytes and lowers the phagocytic activity of neutrophils. In addition, in LPS-stimulated neutrophils it weakens the expression of CD11 and CD16 surface receptors, affecting this way their chemotaxis and adhesion. ${ }^{49}$

\section{Xylocaine}

Another drug used in OFA is xylocaine, which is generally used as a local anesthetic.

The analgesic effect of xylocaine is associated with blockade of nerve conduction, including nociceptive stimuli, due to sodium channel blockage. In relation to the immune system, xylocaine has anti-inflammatory effects by blocking the EGF receptor, inhibiting IL-1 release, and improving the cytotoxic activity of neutrophils. ${ }^{59,60}$

Undoubted benefits of xylocaine include reduction of pain intensity and demand for opioids and lowering the risk of intestinal atony and vomiting, especially in patients after abdominal surgery. ${ }^{61}$

\section{NSAIDs}

Nonsteroidal anti-inflammatory drugs (NSAIDs) participate in pain control and inflammation reduction due to their analgesic potency and anti-inflammatory effects. NSAIDs act by inhibition of the cyclooxygenase (COX) enzyme pathway and synthesis of prostaglandins that play key roles in trauma response. ${ }^{62}$ The positive response to NSAIDs is not only related to their analgesic and anti-inflammatory effects. For example, they can also promote the process of healing in tissues by stimulation of collagen synthesis. ${ }^{63}$

NSAIDs can also disturb immune homeostasis within the gastrointestinal mucosa. The results of a study on animal model presented by Maseda et al has shown an imbalance in the synthesis of the PG and damage to the intestinal barrier resulting in bacterial penetration into the blood vessels due to short-term oral dose of indomethacin. These symptoms are connected with dysregulation of the immune responses, intestinal microbiota, and severe histopathologically confirmed intestinal tissue damage. ${ }^{64}$

NSAIDs inhibit neutrophil and macrophage functions. By inhibiting the proliferation and differentiation of B-lymphocytes, NSAIDs can contribute to the reduction of antibody synthesis. In relation to $\mathrm{T}$ lymphocytes, NSAIDs act rather as stimulants of their function. ${ }^{65,66}$ On the other hand, animal studies have shown that the perioperative combination of $\beta$ blockers (propranolol, etodolac) and NSAIDs (COX-2 indomethacin inhibitor, celecoxib) reduce the risk of the spreading of cancer as a result of their weakening of the inhibition of the NK cell toxicity by surgical trauma. ${ }^{67}$ In comparative studies of fentanyl vs a combination of fentanyl and flurbiprofen, it has been shown that when a combination of NSAID with a low dose of fentanyl is used, more than single doses of fentanyl contribute to a decrease in the levels of VEGF-C, TNF- $\alpha$, and IL- $1 \beta$ molecules, which at increased concentrations are associated with higher risk of postoperative metastases. ${ }^{68}$

\section{Dexmedetomidine}

Dexmedetomidine is a complete agonist of $\alpha 2$ receptors for which it has high selectivity. Stimulation of alpha-2-adrenergic receptors changes the activity of ion channels (potassium and calcium) in the cell membrane of spinal cord neurons, the effect of which manifests as the analgesic, hypnotic, and sedative effects of dexmedetomidine. ${ }^{69,70}$ Over $90 \%$ of dexmedetomidine is bound to proteins, after which its metabolism takes place in the liver with the participation of 
isoenzymes of the cytochrome CYP2B6. Ninety percent of their removal occurs through the kidneys. Dexmedetomidine used as a continuous intravenous infusion during anesthesia reduces the need of supplying inhaled anesthetics and opioids. Common side effects include disturbances of glucose concentrations, hypotension, and arrhythmias. ${ }^{71,72}$ Since dexmedetomidine is an adrenoreceptor agonist, it is worth noting that the sympathetic nervous system plays an important role in the autoregulation of the relationship between the CNS and the immune system through the adrenergic receptors.

Like opioid receptors, adrenergic receptors belong to the group of $\mathrm{G}$ receptors and are located on the central and peripheral cells of the lymphatic, immune, and CNS organs, with $\beta$ receptor expression confirmed mainly on the immune response cells and with $\alpha$ receptors also recognized on some of them. ${ }^{73-75}$ Through adrenergic receptors, adrenaline (A) and NA neurotransmitters stimulate monocytes to synthesize the C2-C5 complement factors involved in the activation and regulation of the complement system. The expression of mRNA for $\alpha$ receptors was also confirmed in polymorphonuclear leukocytes (PMN), and although both amines limited the PMN migration through $\beta$ receptors, the effect of adrenaline on the expression of CD11 was observed with the use of an $\alpha$ receptor. $^{76}$

Furthermore, noradrenaline has been shown to influence the migration of dendritic cells and helper lymphocytes via the $\alpha$ and $\beta$ receptors located on the cells. ${ }^{77}$ It has been confirmed that the immune response cells express predominantly the $\beta$ receptors with $\alpha$ receptors also distinguished on some of them. As an example, it has been confirmed that the NK cell expresses predominantly $\beta$ receptors, although it has been shown in an animal model that NA, just like clonidine, increases the NK cytotoxicity via the $\alpha$-receptors. ${ }^{78}$

According to the research conducted by Priyanka and ThyagaRajan on an animal model, activation of the $\alpha$ receptors was contributing to the inhibition of lymphocyte proliferation and to IFN $\gamma$ synthesis by modulating the expression of the signaling pathways ERK, CREB, Akt, and NF- $\mathrm{KB}$ that depend on the $\alpha 1$ or $\alpha 2$ receptor subtype. ${ }^{79}$ The above study results may explain the negative effect of dexmedetomidine on immune modulation. Dexmedetomidine inhibits the maturation and activity of dendritic cells by reducing the expression of I-A (b) and CD86 signaling molecules on their surface. It also limits the proliferation of helper lymphocytes and cytotoxic activity. ${ }^{80,81}$
Perioperative use of dexmedetomidine contributes to a decrease in the intensity of postoperative pain when, as shown by the results of the research by Andjelkovi et al, the analgesic effect of dexmedetomidine lasted shorter than the effect of xylocaine in patients undergoing abdominal surgery. ${ }^{82}$ According to the authors, an undesirable effect of using both drugs was a decrease in bowel function.

It should be emphasized, however, that the results of many studies indicate that the use of dexmedetomidine has reduced the need for analgesics in the postoperative period. ${ }^{83,84}$

Considering the effects of dexmedetomidine in reducing the number of leukocytes and the concentration of proinflammatory cytokines synthesized by monocytes, the effect of dexmedetomidine is predominantly antiinflammatory, which in groups of patients with impaired immune function may be unfavorable in terms of the risk of infection and to wound healing.

Dexmedetomidine like clonidine belongs to group of $\alpha_{2}$-adrenoceptor ( $\alpha_{2}$ AR) agonists.

\section{Clonidine}

Clonidine is an agonist for the imidazoline and the alphaadrenergic class of receptors with stronger affinity for presynaptic $\alpha_{2}$ adrenoceptors ( $\alpha_{2}$ AR) than for $\alpha_{1}$ receptors $\left(\alpha_{1}\right.$ AR).

The action of clonidine via $\alpha_{2}$ AR, located on sympathetic terminals decreased norepinephrine plasma concentration. ${ }^{85,86}$

Clonidine compared to dexmedetomidine has similar sedative and analgesic properties and these $\alpha_{2}$ agonists used in anesthesia have been associated with reduced anesthetic and analgesic requirements. ${ }^{87}$

There is numerous evidence that $\alpha_{2}$ AR agonists have influence on stress reaction in two ways: centrally, they induce analgesia via presynaptic $\alpha_{2}$ AR in the descending pathways and peripherally by stimulation of anti-inflammatory response. ${ }^{88}$ Clonidine also has immunomodulating properties via attenuating the sympathetic activity. The study conducted by von Drossov et al confirmed the immunomodulating properties of clonidine infusion on cellular response in patients who underwent cardiac surgery. ${ }^{85}$

The obtained results showed the significantly lower ratio of Th1/Th2 (helper lymphocytes) and Tc1/Tc2 (cytotoxic lymphocytes) in patient from clonidine group at $6 \mathrm{~h}$ postoperatively, which may indicate a modulating effect of clonidine on lymphocytes $\mathrm{T}$ subpopulations in 
the early postoperative period. The authors did not observe the influence of clonidine on systemic inflammatory response assessed with plasma cytokines TNF- $\alpha$, IL-6, IL-8, and LPS-stimulated.

In turn, Devereaux et al presented the results of POST 2 study applied the impact of low-dose clonidine in the prevention of myocardial infarction in patient with a risk of ischemia who underwent noncardiac surgery. ${ }^{89}$ The obtained results did not confirm the efficacy and safety of clonidine compared with placebo and aspirin in assessed groups of patients. Moreover, the authors observed more incidences of hypotension, bradycardia, and nonfatal cardiac arrest in clonidine group.

The animal study conducted by Forget et al has shown the influence of various analgesics (fentanyl, ketamine, clonidine) on cellular immunity in particular NK cells activity and an expansion of tumor metastases. ${ }^{46}$ The obtained results have shown that either surgery or chosen analgesics can effect on NK cells activity and cancer cells spread. So that, among these chosen drugs, only fentanyl seemed to have stronger depressive effect on NK cells activity and as the only analgesic can provoke a growth metastases.

Both ketamine and clonidine revealed the significant attenuating of NK cells activity and negative impact on metastases spread.

In conclusion, clonidine has positive effects on cardiovascular system, reduces catecholamine release and can be useful for management sedation and pain with minimal risk of respiratory depression unlike the other drugs. With respect to immune response, clonidine does not seem to be a significant drug, particularly when used in low dose under anesthesia. It needs to be highlighted that severe and sometimes fatal side effects like rebound hypertension, tachycardia, and cardiac arrest should be kept in mind.

\section{Key Points}

Ketamine-induced immunosuppression is reflected as a greater risk of the growth of cancer cells, which may constitute a significant contraindication to long-term use of ketamine in cancer patients.

Benefits of xylocaine include reduction of pain intensity, demand for opioids, and lowering the risk of intestinal atony and vomiting.

NSAIDs inhibit neutrophil and macrophage functions. By inhibiting the proliferation and differentiation of B-lymphocytes, NSAIDs can contribute to the reduction of antibody synthesis. A combination of NSAID with a low dose of fentanyl more than single doses of fentanyl can limit the risk of postoperative metastases.

Dexmedetomidine presents the negative predominantly anti-inflammatory which in groups of patients with impaired immune function may be unfavorable in terms of the risk of infection and to wound healing.

With respect to immune response, clonidine does not seem to be a significant drug, particularly when is used in low dose under anesthesia. It needs to be highlighted that severe and sometimes fatal side effects like rebound hypertension, tachycardia, and cardiac arrest should be kept in mind.

It is worth emphasizing that apart from the drugs assigned to these methods of anesthesia, there is a group of common drugs used in both opioid and opioid-free anesthesia, which also leave a mark on the immune system response. Among the drugs and methods of anesthesia that are successfully used both together with opioids and in the OFA method include intravenous, inhalational, and regional anesthetics.

To sum up, it may be acknowledged that among the techniques and drugs used during anesthesia there are none that would not leave their marks on the immune response. Whether their reflection will remain a scar or a memory depends primarily on the condition of the patient's organism, the efficiency of its defensive mechanisms, and its ability to maintain homeostasis, which are the elements of the response to a surgical trauma.

The efficiency of defensive reactions is a reflection of the efficiency of the immune, nervous, and endocrine systems, each of which individually and all together constitute the first line of defense in response to injury. The age-old rule "primum non nocere" particularly relates to cancer patients with significant attenuated immune response to stress surgery when the drugs given perioperatively can be the other factors provoking a growth of metastases. So the old truth still stands, that the choice of anesthesia depends on the patient's condition, the scope of surgery, and the experience of the anesthesiologist, and it should still be followed.

\section{Disclosure}

The authors report no conflicts of interest for this work.

\section{References}

1. Scanzano A, Cosentino M. Adrenergic regulation of innate immunity: a review. Front Pharmacol. 2015;6:171. 
2. Aamri E, Basnawi A. Effects of anesthesia \& anesthetic techniques on cellular immunity. J Anesth Crit Care Open Access. 2017;7 (6):2-8.

3. von Dossow V, Sander M, MacGill M, Spies C. Perioperative cell-mediated immune response. Front Biosci. 2008; 1 (13):3676-3684. doi: $10.2741 / 2958$

4. Gregory J, Tsay GJ, Zouali M. The interplay between innate-like b cells and other cell types in autoimmunity. Semin Immunopathol. 2018;40(4):407-419.

5. Stein C, Hassan AH, Przewłocki R, Gramsch C, Peter K, Herz A. Opioids from immunocytes interact with receptors on sensory nerves to inhibit nociception in inflammation. Proc Natl Acad Sci USA. 1990;87(15):5935-5939. doi:10.1073/pnas.87.15.5935

6. Cabot PJ. Immune-derived opioids and peripheral antinociception. Clin Exp Pharmacol Physiol. 2001;28(3):230-232. doi:10.1046/ j.1440-1681.2001.03425.x

7. Thyaga Rajan S, Priyanka HP. Bidirectional communication between the neuroendocrine system and the immune system: relevance to health and diseases. Ann Neurosci. 2012;19(1):40-46.

8. Fife BT, Pauken KE, Eagar TN. Interactions between programmed death-1 and programmed death ligand-1 promote tolerance by blocking the $\mathrm{T}$ cell receptor-induced stop signal. Nat Immunol. 2009;10 (11):1185-1192. doi:10.1038/ni.1790

9. Lin E, Calvano SE, Lowry SF. Inflammatory cytokines and cell response in surgery. Surgery. 2000;127:117-126. doi:10.1067/ msy.2000.101584

10. Menger MD, Vollmar B. Surgical trauma: hyperinflammation versus Langenbecks. Arch Surg. 2004;389(6):475-484. (). doi:10.1007/ s00423-004-0472-0

11. Liang X, Liu R, Chen C. Opioid system modulates the immune function: a review. Transl Perioper Pain Med. 2016;1(1):5-13.

12. Tabarra I, Soares S, Rosado T, et al. Novel synthetic opioids toxicological aspects and analysis. Forensic Sci Res. 2019;4 (2):111-140. doi:10.1080/20961790.2019.1588933

13. Stanley TH. The fentanyl story. Pain. 2014;15(12):1215-1226.

14. Wilde M, Pichini S, Pacifici R, et al. Metabolic pathways and potencies of new fentanyl analogs. Front Pharmacol. 2019;05.

15. Komatsu R, Turan AM, Orhan-Sungur M, McGuire J, Radke OC, Apfel CC. Remifentanil for general anaesthesia: a systematic review. Anaesthesia. 2007;62(12):1266-1280. doi:10.1111/j.1365-2044.2007.052 21.x

16. Waldhoer M, Bartlett SE, Whistler JL. Opioid receptors. Annu Rev Biochem. 2004;73::953-990. doi:10.1146/annurev.biochem.73.011303. 073940

17. Pasternak G, Pan YX. Mu opioid receptors in pain management. Acta Anest. Taiwan. 2011;49(1):21-25.

18. Pan L, Xu J, Yu R, Xu MM, Pan YX, Pasternak GW. Identification and characterization of six new alternatively spliced variants of the human mu opioid receptor gene, Oprm. Neuroscience. 2005;133 (1):209-220. doi:10.1016/j.neuroscience.2004.12.033

19. Hahn EF, Pasternak GW. Naloxonazine, a potent, long-lasting inhibitor of opiate binding sites. Life Sci. 1982;31(1213):1385-1388. doi:10.1016/0024-3205(82)90387-3

20. Kohno T, Kumamoto E, Higashi H, Shimoji K, Yoshimura M. Actions of opioids on excitatory and inhibitory transmission in substantia gelatinosa of adult rat spinal cord. J Physiol. 1999;518 ((Pt 3)):803-813. doi:10.1111/j.1469-7793.1999.0803p.x

21. Sharp BM. Multiple opioid receptors on immune cells modulate intracellular signaling. Brain Behav Immun. 2006;20(1):9-14. doi:10.1016/j.bbi.2005.02.002

22. Raisch J, Arlette Darfeuille-Michaud A, Nguyen H. Role of microRNAs in the immune system, inflammation and cancer. World J Gastroenterol. 2013;19(20):2985-2996. doi:10.3748/wjg.v19.i20.2985

23. ChK H, Wagley Y, Law P, Wei L, Loh H. microRNAs in opioid pharmacology. J Neuroimmune Pharmacol. 2012;7(4):808-819. doi:10.1007/s11481-011-9323-2
24. O’Brien J, Hayder H, Zayed Y, Peng C. Overview of MicroRNA. Biogenesis, mechanisms of actions, and circulation. Front Endocrinol. 2018;9:402. doi:10.3389/fendo.2018.00402

25. O'Connell RM, Dinesh S, Baltimore R, Baltimore D. microRNA regulation of inflammatory responses. Annu Rev Immunol. 2012;30:295-312. doi:10.1146/annurev-immunol-020711-075013

26. Liston A, Papadopoulou AS, Danso-Abeam D, Dooley J. MicroRNA29 in the adaptive immune system: setting the threshold. Cell Mol Life Sci. 2012;69(21):3533-3541. doi:10.1007/s00018-012-1124-0

27. Zhang Q, Cannavicci A, Dai SC, Kurtyk M. MicroRNA signature of human blood mononuclear cells. Molecular Cell Biochem. 2019;462 (12):167-172. doi:10.1007/s11010-019-03619-3

28. Tsitsiou E, Lindsay MA. microRNAs and the immune response. Curr Opin Pharmacol. 2009;9(4):514-520. doi:10.1016/j.coph.2009.05.003

29. Lindsay MA. microRNAs and the immune response. Trends Immunol. 2008;29(7):343-351. doi:10.1016/j.it.2008.04.004

30. Zheng H, Zeng Y, Zhang X, Chu J, Loh H, Law P. \{micro\}-Opioid receptor agonists differentially regulate the expression of miR-190 and NeuroD. Mol Pharmacol. 2010;77:102-109. doi:10.1124/ mol.109.060848

31. Zheng H, Chu J, Zeng Y, et al. 1 phosphorylation contributes to the differential effects of mu-opioid receptor agonists on microRNA-190 expression. J Biol Chem. 2010a;285(29):21994-22002. doi:10.1074/ jbc.M110.112607

32. Ni J, Gao Y, Gong S, Guo S, Hisamisu T, Jiang X. Regulation of $\mu$ opioid type 1 receptors by microRNA134 in dorsal root ganglion neurons following peripheral inflammation. EJP. 2013;7(3):313-323. doi:10.1002/j.1532-2149.2012.00197.x

33. Börner C, Lanciotti S, Koch T, Höllt V, Kraus J. mu opioid receptor agonist-selective regulation of interleukin-4 in $\mathrm{T}$ lymphocytes. $J$ Neuroimmunol. 2013;263(1-2):35-42. doi:10.1016/j.jneuroim. 2013.07.012

34. Lisowska B, Szymańska M, Nowacka E, Olszewska M. Anesthesiology and the cytokine network. Postepy Hig Med Dosw. 2013;67:761-769. doi:10.5604/17322693.1061412

35. De Cosmo G, Sessa F, Fiorini F, Congedo E. Effect of remifentanil and fentanyl on postoperative cognitive function and cytokines level in elderly patients undergoing major abdominal surgery. J Clin Anesth. 2016;35:40-46. doi:10.1016/j.jclinane.2016.07.016

36. von Dossow V, Luetz A, Haas A, et al. Effects of remifentanil and fentanyl on the cell-mediated immune response in patients undergoing elective coronary artery bypass graft surgery. J Int Med Res. 2008;36:1235-1247. doi:10.1177/147323000803600610

37. Bidlack JM. Detection and function of opioid receptors on cells from the immune system. Clin Diagn Lab Immunol. 2000;7(5):719-723. doi:10.1128/CDLI.7.5.719-723.2000

38. Jacobs R, Karst M, Scheinichen D, et al. Effects of fentanyl on cellular immune functions in man. Int $J$ Immunopharmacol. 1999;21(7):445-454. doi:10.1016/S0192-0561(99)00025-9

39. Li ZH, Chu N, Shan LD, Gong S, Yin QZ, Jiang XH. Inducible expression of functional $\mathrm{mu}$ opioid receptors in murine dendritic cells. J Neuroimmune Pharmacol. 2009;4(3):359-367. doi:10.1007/ s11481-009-9145-7

40. Kortylewski M, Xin H, Kujawski M, et al. Regulation of the IL-23 and IL-12 balance by Stat3 signaling in the tumor microenvironment. Cancer Cell. 2009;15(2):114-123. doi:10.1016/j.ccr.2008.12.018

41. Miao J, Wang L, Chen L, Yang T, Jin L, Lin L. Fentanyl inhibits cell viability in human pancreatic cancer cell line and tumor growth in pancreatic cancer cell-transplanted mice. Int J Clin Exp Med. 2015;8 (10):17684-17693.

42. Kim R. Effects of surgery and anesthetic choice on immunosuppression and cancer recurrence. J Transl Med. 2018;16(8):1-13. doi:10.1186/s12967-018-1389-7

43. Dang Y, Shi X, William XW, Zuo M. The effect of anesthesia on the immune system in colorectal cancer patients. Can J Gastroenterol Hepatol. 2018;1-9. 
44. Yardeni IZ, Beilin B, Mayburd E, Alcalay Y, Bessler H. Relationship between fentanyl dosage and immune function in the postoperative period. J Opioid Manag. 2008;4(1):27-33. doi:10.5055/jom.2008.0005

45. Beilin B, Shavit Y, Hart J, et al. Effects of anesthesia based on large versus small doses of fentanyl on natural killer cell cytotoxicity in the perioperative period. Anesth Analg. 1996;82(3):492-497.

46. Forget P, Collet V, Lavand'homme P, De Kock M. Does analgesia and condition influence immunity after surgery? Effects of fentanyl, ketamine and clonidine on natural killer activity at different ages. Eur $J$ Anaesthesiol. 2010;27(3):233-240. doi:10.1097/EJA.0b013e32832 $\mathrm{d} 540 \mathrm{e}$

47. Sultana A, Torres D, Schumann R. Special indications for opioid free anaesthesia and analgesia, patient and procedure related: including obesity, sleep apnoea, chronic obstructive pulmonary disease, complex regional pain syndromes, opioid addiction and cancer surgery. best practise and research. Clinical Anesthesiology. 2017;31 (4):547-560.

48. Boysen PG, Pappas MM, Evans B. An evidence-based opioid-free anesthetic technique to manage perioperative and periprocedural pain. Ochsner J. 2018;18(2):121-125. doi:10.31486/toj.17.0072

49. Liu FL, Chen TL, Chen RM. Mechanisms of ketamine-induced immunosuppression. Acta Anaesthesiologica Taiwanica. 2012;50:172-177. doi:10.1016/j.aat.2012.12.001

50. Beilin B, Rusabrov Y, Shapira Y, et al. Low-dose ketamine affects immune responses in humans during the early postoperative period. Br J Anaesth. 2007;99(4):522-527. doi:10.1093/bja/aem218

51. Roytblat L, Roy-Shapira A, Geemberg L, et al. Preoperative low dose ketamine reduces serum interleukin-6 response after abdominal hysterectomy. Pain Clin. 1996;9:327-334.

52. Kawasaki T, Ogata M, Kawasaki C, Ogata J, Inoue Y, Shigematsu A. Ketamine suppresses proinflammatory cytokine production in human whole blood in vitro. Anesth Analg. 1999;89(3):665-669.

53. Jimi N, Segawa K, Minami K, Sata T, Shigematsu A. Inhibitory effect of the intravenous anesthetic, ketamine, on rat mesangial cell proliferation. Anesth Analg. 1997;84(1):190-195. doi:10.1213/ 00000539-199701000-00034

54. Li Y, Shen R, Wen G, et al. Effects of ketamine on levels of inflammatory cytokines IL-6, IL-1, and TNF- in the hippocampus of mice following acute or chronic administration. Front Pharmacol. 2017;8:139.

55. Melamed R, Bar-Yosef S, Shakhar G, Shakhar K, Ben-Eliyahu S. Suppression of natural killer cell activity and promotion of tumor metastasis by ketamine, thiopental, and halothane, but not by propofol: mediating mechanisms and prophylactic measures. Anesth Analg. 2003;97(5):1331-1339. doi:10.1213/01.ANE.0000082995.44040.07

56. Loftus RW, Yeager MP, Clark JA, et al. Intraoperative ketamine reduces perioperative opiate consumption in opiate-dependent patients with chronic back pain undergoing back surgery. Anesthesiology. 2010;113:639-646. doi:10.1097/ALN.0b013e3181e90914

57. Kamp J, Van Velzen M, Olofsen E. Pharmacokinetic and pharmacodynamic considerations for NMDA-receptor antagonist ketamine in the treatment of chronic neuropathic pain: an update of the most recent literature. Expert Opin Drug Metab Toxicol. 2019;15 (12):1033-1041. doi:10.1080/17425255.2019.1689958.

58. Dong TT, Mellin-Olsen J, Gelb AW. Ketamine: a growing global health-care need. Br J Anaesth. 2015;115(4):491-493.

59. Yardeni IZ, Beilin B, Mayburd E. The effect of perioperative intravenous lidocaine on postoperative pain and immune function. Anesth Analg. 2009;109(5):1464-1469. doi:10.1213/ANE.0b013e3181bab $1 \mathrm{bd}$

60. Puig NR, Ferrero P, Bay ML, et al. Effects of sevoflurane general anesthesia: immunological studies in mice. Int Immunopharmacol. 2002;2(1):95-104. doi:10.1016/S1567-5769(01)00151-5

61. Gaszynski T. Mozanski M: recommendations of perioperative care and general anesthesia (including low and free opioid anesthesia) for the obese patient. Anest Ratow. 2016;10:67-77.
62. Lisowska B, Kosson D, Domaracka K. Lights and shadows of NSAIDs in bone healing: the role of prostaglandins in bone metabolism. Drug Des Devel Ther. 2018;121753-121758.

63. Lisowska B, Kosson D, Domaracka K. Positives and negatives of nonsteroidal anti-inflammatory drugs in bone healing: the effects of these drugs on bone repair. Drug Des Devel Ther. 2018;12:1809-1814. doi:10.2147/DDDT.S164565

64. Maseda D, Zackular JP, Trindade B, et al. Nonsteroidal anti-inflammatory drugs alter the microbiota and exacerbate Clostridium difficile colitis while dysregulating the inflammatory response. $m$ Bio. 2019;10(1).

65. Cortet B, Duquesnoy B. Action of non-steroidal anti-inflammatory agents on the immune system. Rev Rhum Mal Osteoartic. 1991;58 (5):379-386.

66. Bancos S, Bernard MP. Ibuprofen and other widely used non-steroidal anti-inflammatory drugs inhibit antibody production in human cells. Cell Immunol. 2009;258(1):18-28. doi:10.1016/j.cellimm.2009.03.007

67. Benish M, Bartal I, Goldfarb Y. Perioperative use of beta-blockers and COX-2 inhibitors may improve immune competence and reduce the risk of tumor metastasis. Ann Surg Oncol. 2008;15(7):2042-2052. doi:10.1245/s10434-008-9890-5

68. Wen Y, Wang M, Yang J, et al. A comparison of fentanyl and flurbiprofen axetil on serum VEGF-C, TNF- $\alpha$, and IL-1ß concentrations in women undergoing surgery for breast cancer. Pain Pract. 2015;15(6):530-537. doi:10.1111/papr.12206

69. Eisenach JC, De Kock M. Alpha2 adrenergic agonists for regional anesthesia: A clinical review of clonidine (19841995). Anesthesiology. 1996;85:655-674. doi:10.1097/00000542-199609000-00026

70. Kamibayashi T, Maze M. Perioperative use of alfa-2 adrenergic agonists. Curr Opinion Anaesth. 1996;9:323-327. doi:10.1097/ 00001503-199608000-00011

71. Maze M, Tranquilli W. Alpha-2 adrenoceptor agonists: defining the role in clinical anesthesia. Anesthesiology. 1991;74:581-605. doi:10.1097/00000542-199103000-00029

72. Hall JE, Uhrich TD, Barney JA, Arain S, Ebert TJ. Sedative, amnestic, and analgesic properties of small-dose dexmedetomidine infusions. Anesth Analg. 2000;90:699-705. doi:10.1097/00000539-20000300000035

73. Elenkov IJ, Wilder RL, Chrousos GP, Vizi ES. The sympathetic nerve-an integrative interface between two supersystems: the brain and the immune system. Pharmacol Rev. 2000;52(4):595-638.

74. Roggero E, Besedovsky HO, Del Rey A. The role of the sympathetic nervous system in the thymus in health and disease. Neuroimmunomodulation. 2011;18(5):339-349. doi:10.1159/000329581

75. Scanzano A, Cosentino M. Adrenergic regulation of innate immunity: a review. Front Pharmacol. 2015;13(6):171.

76. Scanzano A, Schembri L, Rasini E, et al. Adrenergic modulation of migration, CD11b and CD18 expression, ROS and interleukin- 8 production by human polymorphonuclear leukocytes. Inflamm Res. 2015;64(2):127-135. doi:10.1007/s00011-014-0791-8

77. Maestroni GJ. Modulation of skin norepinephrine turnover by allergen sensitization: impact on contact hypersensitivity and $\mathrm{T}$ helper priming. J Invest Dermatol. 2004;122(1):119-1124. doi:10.1046/ j.0022-202X.2003.22132.x

78. Xiao J, Huang HW, Peng YP, Bao JY, Huang Y, Qiu YH. Modulation of natural killer cell function by alpha-adrenoreceptor-coupled signalling. Neuro Endocrinol Lett. 2010;31(5):635-644.

79. Priyanka HP, ThyagaRajan S. Selective modulation of lymphoproliferation and cytokine production via intracellular signaling targets by $\alpha 1$ - and $\alpha 2$-adrenoceptors and estrogen in splenocytes. Int Immunopharmacol. 2013;17(3):774-784. doi:10.1016/j.intimp.2013.08.020

80. Ueshima H, Inada T, Shingu K. Suppression of phagosome proteolysis and Matrigel migration with the $\alpha 2$-adrenergic receptor agonist dexmedetomidine in murine dendritic cells. Immunopharmacol Immunotoxicol. 2013;35(5):558-566. doi:10.3109/08923973.2013.82 2509 
81. Chen G, Le Y, Zhou L, et al. Dexmedetomidine inhibits maturation and function of human cord blood-derived dendritic cells by interfering with synthesis and secretion of IL-12 and IL-23. PLoS One. 2016;11(4)

82. Andjelkovi L, Novak-Jankovi V, Pozar-Lukanovi N, Bosnić Z, Spindler-Vesel A. Influence of dexmedetomidine and lidocaine on perioperative opioid consumption in laparoscopic intestine resection: a randomized controlled clinical trial. J International Medical Research. 2018;46(12):5143-5154. doi:10.1177/0300060518792456

83. Grape S, Kirkham KR, Frauenknecht J, Albrecht E. Intra-operative analgesia with remifentanil vs. dexmedetomidine: a systematic review and meta-analysis with trial sequential analysis. Anaesthesia 2019;74(6):793-800. doi:10.1111/anae.14657

84. Singh PM, Panwar R, Borle A, Mulier JP, Sinha A, Goudra B. Perioperative analgesic profile of dexmedetomidine infusions in morbidly obese undergoing bariatric surgery: a meta-analysis and trial sequential analysis. Surg Obes Relat Dis. 2017;13(8):1434-1446. doi:10.1016/j.soard.2017.02.025
85. von Dossow V, Cand NB, Moshirzadeh M, et al. Clonidine attenuated early proinflammatory response in t-cell subsets after cardiac surgery. Anest Analg. 2006;103:809-814. doi:10.1213/01.ane.0000237308. 28739.d8

86. Lv Q, Wu F, Gan X, et al. The involvement of descending pain inhibitory system in electroacupuncture-induced analgesia. Front Integr Neurosci. 2019;13:38. doi:10.3389/fnint.2019.00038

87. Ebert TJ, Hall JE, Barney JA, Uhrich TD, Colinco MD. The Effects of Increasing Plasma Concentrations of Dexmedetomidine in Humans. Anesthesiology. 2000;93:382-394. doi:10.1097/00000542200008000-00016

88. Giovannitti JA, Thoms SM, Crawford JJ. Alpha-2 adrenergic receptor agonists: a review of current clinical applications. Anesth Prog. 2015;62(1):31-38. doi:10.2344/0003-3006-62.1.31

89. Devereaux PJ, Sessler DI, Leslie K, et al. Clonidine in patients undergoing noncardiac surgery. $N$ Engl J Med. 2014;370:1504-1513. doi:10.1056/NEJMoa1401106
Journal of Inflammation Research

\section{Publish your work in this journal}

The Journal of Inflammation Research is an international, peerreviewed open-access journal that welcomes laboratory and clinical findings on the molecular basis, cell biology and pharmacology of inflammation including original research, reviews, symposium reports, hypothesis formation and commentaries on: acute/chronic inflammation; mediators of inflammation; cellular processes; molecular

\section{Dovepress}

mechanisms; pharmacology and novel anti-inflammatory drugs; clinical conditions involving inflammation. The manuscript management system is completely online and includes a very quick and fair peerreview system. Visit http://www.dovepress.com/testimonials.php to read real quotes from published authors. 